\title{
Effect of Different Plant Growth Regulators, Micronutrients and Photothermal Regimes on Growth, Phenological and Yield Characters of Brinjal (Solanum melongena L.)
}

\author{
Diksha Manaware* and D.P. Sharma \\ Department of Horticulture, Jawaharlal Nehru Krishi Vishwa Vidyalaya, \\ Jabalpur (M.P.), India \\ *Corresponding author
}

\section{A B S T R A C T}

\begin{tabular}{l} 
Ke y w o r d s \\
Photothermal \\
Regimes, \\
Brinjal, \\
Solanum melongena \\
\hline Article Info \\
$\begin{array}{l}\text { Accepted: } \\
\text { 26 September } 2020 \\
\text { Available Online: } \\
\text { 10 October } 2020\end{array}$ \\
\hline
\end{tabular}

An investigation entitled "Effect of different plant growth regulators, micronutrients and photothermal regimes on morphological and phenological characters of Brinjal (Solanum melongena L.)" was conducted at Horticulture Complex, Department of Horticulture, College of Agriculture JNKVV, Jabalpur (M.P.) during the year 2018-19. The experiment consists of forty five treatments comprising plant growth regulators, micronutrients and different photothermal regimes and was laid out in randomized block design having three replications. There were two plant growth regulators Brassinosteroids $(0.5 \mu \mathrm{M}, 1.0 \mu \mathrm{M})$, $\mathrm{GA}_{3}(25 \mathrm{ppm}, 50 \mathrm{ppm})$ and two micronutrients Boron (100 ppm), Molybdenum $(2.0 \mu \mathrm{mol} / \mathrm{l})$ were use with different combinations and were applied by foliar application on $15^{\text {th }}$ November, $30^{\text {th }}$ November and $15^{\text {th }}$ December to assess the effect on growth and yield of brinjal. The investigation revealed that, the vegetative growth observations (plant height of plant at 30, 60 and 90 DAT, number of primary branches per plant and number of flower per cluster), phenological observations (viz. minimum days to flower initiation, days to $50 \%$ flowering, days to first picking) and yield per plot was reported in treatment where plant has been sprayed with $\mathrm{T}_{9}\left(\mathrm{GA}_{3}(50 \mathrm{ppm})+\right.$ Boron $(100 \mathrm{ppm})$ whereas the control was lowest.

\section{Introduction}

Brinjal (Solanum melongena L.) also known as eggplant in USA and aubergine in France and UK is a member of angiospermic family Solanaceae. It is known as King of Vegetables. It is a popular vegetable crop widely grown in tropics and subtropics (Roychowdhury and Tah, 2011). According to Vavilov (1928), the eggplant originated in Indo-Burma region. India is the primary centre of origin (Zeven and Zhukovsky, 1975) while secondary diversity in China and South East Asia (Nath et al., 1987).

India is the second largest producer of brinjal in the world. Area under brinjal in India is 730 thousand hectare with production of 12801 thousand metric tonnes and productivity is 17.5 metric tonnes/ hectare. It is grown in 51.35 thousand hectare area in Madhya Pradesh with a total annual 
production of 1073.63 thousand metric tonnes with 20.19 metric tonnes/hectare productivity (National Horticulture Board, 2018). Brinjal contains 92.7 per cent water, 4 percent carbohydrates, 1.4 per cent protein, 1.3 per cent fiber, 0.3 per cent fats, 0.3 per cent minerals and vitamin $\mathrm{A}$ in a negligible quantity (Tindall, 1978) and it is also a rich source of minerals like potassium, calcium, sodium and iron (Mohamed et al., 2003; Raigon et al., 2008) as well as dietary fibre (USDA, 2014; Sanchez-Castillo et al., 1999). Brinjal is an annual herbaceous plant. Inflorescence is often solitary but sometimes it constitutes a cluster of 2-5 flowers. Flower is complete, actinomorphic and hermaphrodite. Only long and medium styled flowers are considered as fruit setting flowers. Further, possibilities of cross pollination are more in long style flowers. Fruit setting of long styled flowers ranges from $70 \%$ to $86.7 \%$ in different varieties. In medium styled flowers, fruit set varies from $12.5 \%$ to $55.6 \%$.

Climate change results in crop failures, reduction in yield and quality and increasing pest and disease problems which renders the vegetable cultivation unprofitable. Brinjal is a warm season crop and susceptible to severe frost. Climatic conditions, especially low temperature during cool season cause abnormal development of the ovary (splitting) in flower buds which then differentiate and develop into deformed fruits during that season (Nothmann and koller, 1973). Cool nights and short summers are unfavorable to its satisfactory yield. The reduction in the yield of brinjal is due to to its poor physiological efficiency, poor fruit setting and non- synchronize maturity.

Application of plant growth regulators (PGRs) may play an important role in proper flowering, fruit setting, synchronize maturity, ripening and thereby increase the yield of the crops. Gibberellin promotes shoot growth by accelerating the cell elongation and also increases plant height, number of branches per plant, size of leaves and fruits. (Dhakar and Singh, 2015) and significantly reduces the number of seeds per fruit. Brassinosteroids (BRs) are a novel group of phyto- hormones occurring in plant steroid hormones and are distributed throughout the plant kingdom (Krishna, 2003; Montoya et al., 2005). Brassinosteroids plays prominent roles in various physiologic processes, like cell elongation, pollen tube growth, root inhibition, ethylene biosynthesis, senescence, photosynthesis, and enzyme activation (Sasse 2003, Bajguz and Hayat 2009, Hayat et al., 2012) and it is also have ameliorative effect on plants subjected to environmental stress such as cold stress (Liu et al., 2009), heat stress (Ogweno et al., 2008), oxidative damage (Cao et al., 2005) and pathogen infection (Nakashita et al., 2003; Zhou et al., 2004). It is also observed that application of micronutrients plays a role in improving the yield and quality of brinjal. Boron changes the chemical composition, structure of cell walls, and phenol metabolism and has prominent role in sugar transport, impairment of plasma membrane and phyto hormone metabolism. Molybdenum (Mo) is an essential trace element for plant growth, development and production (Sabatino et al., 2019). It is required for the formation of the nitrate reductase enzyme and has a striking effect on pollen formation (Siddiky et al., 2007). Stress resistance in Molybdenum deficient plants is decreased against low temperature.

\section{Materials and Methods}

The experiment was conducted at Horticulture complex, Department of Horticulture, Jawaharlal Nehru Krishi Vishwa Vidyalaya, Jabalpur (M.P.) during the year 2018-19. The soil of the experimental field was medium black and good drainage uniform texture. The 
experiment was laid out in Randomized Complete Block Design (RCBD- factorial) with three replications. The field experiment consisted of 45 treatments involving the combination of plant growth regulators, micronutrients and different photothermal regimes. Plant growth regulators applied were Brassinosteroids $(0.5 \mu \mathrm{M}, 1.0 \mu \mathrm{M})$ and $\mathrm{GA}_{3}$ (25 ppm, $50 \mathrm{ppm}$ )and micronutrients applied were boron (100 ppm) and molybdenum $(2.0 \mu \mathrm{mol} / \mathrm{l})$ as foliar spray at pre flowering and post flowering stages of plant on three different date of transplanting $\left(15^{\text {th }}\right.$ November, $30^{\text {th }}$ November and $15^{\text {th }}$ December). The observations were recorded in each plot from randomly selected five tagged plant. The recorded observations were plant height at 30, 60, 90 DAT (days after transplanting), number of primary branches per plant, number of flower per cluster, days to flower initiation, days to $50 \%$ flowering, days to first picking and yield $(\mathrm{kg} / \mathrm{plot})$ (Table $1)$.

\section{Results and Discussion}

\section{Plant height at 30, 60 and 90 DAT}

The maximum plant height at 30 DAT was observed in $\mathrm{D}_{1} \mathrm{~T}_{9}$ (47.15) which was transplanted on $15^{\text {th }}$ November with the foliar application of $\mathrm{GA}_{3}(50 \mathrm{ppm})+$ Boron (100ppm) followed by $\mathrm{D}_{1} \mathrm{~T}_{8}(46.62)$ while the minimum plant height at 30 DAT was recorded in control- $\mathrm{D}_{3} \mathrm{~T}_{31}$ (34.26) which was transplanted on $15^{\text {th }}$ December. The maximum plant height at 60 DAT was recorded in $\mathrm{D}_{1} \mathrm{~T}_{9}$ (61.29) which were transplanted on $15^{\text {th }}$ November with the foliar application of $\mathrm{GA}_{3}$ (50ppm) + Boron (100ppm) followed by $\mathrm{D}_{2} \mathrm{~T}_{24}$ (61.19) while the minimum plant height at 60 DAT was recorded in control- $\mathrm{D}_{3} \mathrm{~T}_{31}$ (41.81). The maximum plant height at 90 DAT was recorded in $\mathrm{D}_{1} \mathrm{~T}_{9}$ (76.93) which were transplanted on $15^{\text {th }}$ November with the foliar application $\mathrm{GA}_{3}(50 \mathrm{ppm})+$ Boron (100ppm) of followed by $\mathrm{D}_{2} \mathrm{~T}_{24}$ (75.59) while the minimum plant height at 90 DAT was recorded in control- $\mathrm{D}_{3} \mathrm{~T}_{31}(50.16)$.

The increase in plant height due to the reason that, $\mathrm{GA}_{3}$ stimulate cell division, cell elongation and cell enlargement and ultimately lead to better plant growth and role of boron in cell wall formation, cell development and elongation. The findings of Kumar et al., (2014), El- Gawad and Osman (2014), Islam (2015), Akand et al., (2016), Chauhan et al., (2017) and Jakhar et al., (2018) were similar to that of the present findings (Table 2).

Table.1 Details of treatment

\begin{tabular}{|c|c|c|c|c|c|}
\hline $\mathbf{T}_{1}$ & Control & $\mathbf{T}_{6}$ & Boron (100ppm) & $\mathbf{T}_{11}$ & $\begin{array}{c}\mathrm{GA}_{3}(50 \mathrm{ppm})+\mathrm{Molybdenum} \\
(2.0 \mu \mathrm{mol} / \mathrm{l})\end{array}$ \\
\hline $\mathbf{T}_{\mathbf{2}}$ & $\mathrm{GA}_{3}(25 \mathrm{ppm})$ & $\mathrm{T}_{7}$ & Molybdenum $(2.0 \mu \mathrm{mol} / \mathrm{l})$ & $\mathrm{T}_{12}$ & $\begin{array}{l}\text { Brassinosteroids } \quad(0.5 \mu \mathrm{M})+\text { Boron } \\
(100 \mathrm{ppm})\end{array}$ \\
\hline $\mathbf{T}_{3}$ & $\mathrm{GA}_{3}(50 \mathrm{ppm})$ & $\mathrm{T}_{8}$ & (25ppm)+Boron & $\mathrm{T}_{13}$ & $\begin{array}{l}\text { Brassinosteroids } \\
(1.0 \mu \mathrm{M})+\text { Boron }(100 \mathrm{ppm})\end{array}$ \\
\hline $\mathbf{T}_{4}$ & $\begin{array}{l}\text { Brassinosteroids } \\
(0.5 \mu \mathrm{M})\end{array}$ & $\mathrm{T}_{9}$ & $\begin{array}{l}\mathrm{GA}_{3} \\
(50 \mathrm{ppm})+\operatorname{Boron}(100 \mathrm{ppm})\end{array}$ & $\mathrm{T}_{14}$ & $\begin{array}{l}\text { Brassinosteroids }(0.5 \mu \mathrm{M}) \\
+ \text { Molybdenum }(2.0 \mu \mathrm{mol} / \mathrm{l})\end{array}$ \\
\hline $\mathbf{T}_{5}$ & $\begin{array}{l}\text { Brassinosteroids } \\
(1.0 \mu \mathrm{M})\end{array}$ & $\mathrm{T}_{10}$ & $\begin{array}{l}\mathrm{GA}_{3}(25 \mathrm{ppm})+\text { Molybdenum } \\
(2.0 \mu \mathrm{mol} / \mathrm{l})\end{array}$ & $\mathrm{T}_{15}$ & $\begin{array}{l}\text { Brassinosteroids } \\
+ \text { Molybdenum }(2.0 \mu \mathrm{mol} / \mathrm{l})\end{array}$ \\
\hline
\end{tabular}

1. Three Dates of transplanting at 15 days interval $\left(D_{1}, D_{2}\right.$ and $\left.D_{3}\right)$

2. Plant growth regulators

3. Micronutrients 
Table.2 Effect of various plant growth regulators, micronutrients and photothermal regimes on plant height of brinjal at 30,60, 90 and 120 DAT

\begin{tabular}{|c|c|c|c|c|}
\hline & \multicolumn{3}{|c|}{ Plant height $(\mathrm{cm})$ at } \\
\hline \multicolumn{2}{|c|}{ Treat. Symb. Treatments } & 30 DAT & & 90DAT \\
\hline$T_{1}$ & D1 +Control & 36.65 & 42.88 & 50.71 \\
\hline $\mathbf{T}_{2}$ & $\mathrm{D} 1+\mathrm{GA}_{3}(25 \mathrm{ppm})$ & 42.74 & 50.51 & 60.97 \\
\hline $\mathbf{T}_{3}$ & $\mathrm{D} 1+\mathrm{GA}_{3}(50 \mathrm{ppm})$ & 43.62 & 52.37 & 63.38 \\
\hline $\mathbf{T}_{4}$ & D1 +Brassinosteroids $(0.5 \mu \mathrm{M})$ & 42.89 & 50.35 & 59.48 \\
\hline $\mathbf{T}_{5}$ & D1 +Brassinosteroids $(1.0 \mu \mathrm{M})$ & 43.49 & 50.97 & 60.28 \\
\hline$T_{6}$ & D1 + Boron (100ppm) & 42.35 & 50.64 & 60.60 \\
\hline $\mathbf{T}_{7}$ & D1 + Molybdenum $(2.0 \mu \mathrm{mol} / \mathrm{l})$ & 37.38 & 44.79 & 53.71 \\
\hline $\mathbf{T}_{8}$ & $\mathrm{D} 1+\mathrm{GA}_{3}(25 \mathrm{ppm})+$ Boron $(100 \mathrm{ppm})$ & 46.62 & 58.81 & 74.00 \\
\hline$T_{9}$ & $\mathrm{D} 1+\mathrm{GA}_{3}(50 \mathrm{ppm})+$ Boron(100ppm) & 47.15 & 61.29 & 76.93 \\
\hline$T_{10}$ & $\mathrm{D} 1+\mathrm{GA}_{3}(25 \mathrm{ppm})+$ Molybdenum $(2.0 \mu \mathrm{mol} / \mathrm{l})$ & 42.06 & 56.34 & 70.14 \\
\hline$T_{11}$ & $\mathrm{D} 1+\mathrm{GA}_{3}(50 \mathrm{ppm})+$ Molybdenum $(2.0 \mu \mathrm{mol} / \mathrm{l})$ & 42.17 & 55.68 & 68.26 \\
\hline $\mathbf{T}_{12}$ & D1 +Brassinosteroids $(0.5 \mu \mathrm{M})+$ Boron $(100 \mathrm{ppm})$ & 44.34 & 58.27 & 71.42 \\
\hline $\mathbf{T}_{13}$ & D1 +Brassinosteroids $(1.0 \mu \mathrm{M})+$ Boron $(100 \mathrm{ppm})$ & 44.36 & 60.48 & 72.66 \\
\hline$T_{14}$ & $\mathrm{D} 1+$ Brassinosteroids $(0.5 \mu \mathrm{M})+$ Molybdenum $(2.0 \mu \mathrm{mol} / \mathrm{l})$ & 41.63 & 53.47 & 67.09 \\
\hline $\mathbf{T}_{15}$ & D1 +Brassinosteroids $(1.0 \mu \mathrm{M})+$ Molybdenum $(2.0 \mu \mathrm{mol} / \mathrm{l})$ & 41.74 & 52.98 & 67.28 \\
\hline$T_{16}$ & D2 +Control & 35.92 & 42.36 & 51.16 \\
\hline $\mathbf{T}_{17}$ & $\mathrm{D} 2+\mathrm{GA}_{3}(25 \mathrm{ppm})$ & 41.16 & 49.11 & 58.93 \\
\hline $\mathbf{T}_{18}$ & $\mathrm{D} 2+\mathrm{GA}_{3}(50 \mathrm{ppm})$ & 42.93 & 51.88 & 62.00 \\
\hline$T_{19}$ & $\mathrm{D} 2+$ Brassinosteroids $(0.5 \mu \mathrm{M})$ & 42.23 & 49.51 & 57.96 \\
\hline $\mathbf{T}_{20}$ & D2 +Brassinosteroids $(1.0 \mu \mathrm{M})$ & 43.18 & 51.41 & 61.19 \\
\hline $\mathbf{T}_{21}$ & D2 + Boron (100ppm) & 41.73 & 50.14 & 59.57 \\
\hline $\mathbf{T}_{22}$ & $\mathrm{D} 2+$ Molybdenum $(2.0 \mu \mathrm{mol} / \mathrm{l})$ & 36.47 & 43.91 & 52.36 \\
\hline $\mathbf{T}_{23}$ & $\mathrm{D} 2+\mathrm{GA}_{3}(25 \mathrm{ppm})+$ Boron $(100 \mathrm{ppm})$ & 45.91 & 59.22 & 72.90 \\
\hline $\mathbf{T}_{24}$ & $\mathrm{D} 2+\mathrm{GA}_{3}(50 \mathrm{ppm})+$ Boron(100ppm) & 46.68 & 61.19 & 75.59 \\
\hline $\mathbf{T}_{25}$ & $\mathrm{D} 2+\mathrm{GA}_{3}(25 \mathrm{ppm})+$ Molybdenum $(2.0 \mu \mathrm{mol} / \mathrm{l})$ & 43.86 & 55.45 & 69.21 \\
\hline $\mathbf{T}_{26}$ & $\mathrm{D} 2+\mathrm{GA}_{3}(50 \mathrm{ppm})+$ Molybdenum $(2.0 \mu \mathrm{mol} / \mathrm{l})$ & 41.64 & 55.07 & 66.85 \\
\hline $\mathbf{T}_{27}$ & D2 +Brassinosteroids $(0.5 \mu \mathrm{M})+$ Boron $(100 \mathrm{ppm})$ & 42.73 & 54.66 & 65.97 \\
\hline $\mathbf{T}_{28}$ & $\mathrm{D} 2+$ Brassinosteroids $(1.0 \mu \mathrm{M})+$ Boron $(100 \mathrm{ppm})$ & 43.39 & 56.07 & 69.79 \\
\hline $\mathbf{T}_{29}$ & $\mathrm{D} 2+$ Brassinosteroids $(0.5 \mu \mathrm{M})+$ Molybdenum $(2.0 \mu \mathrm{mol} / \mathrm{l})$ & 41.59 & 54.26 & 67.25 \\
\hline $\mathbf{T}_{30}$ & D2+Brassinosteroids $(1.0 \mu \mathrm{M})+$ Molybdenum $(2.0 \mu \mathrm{mol} / \mathrm{l})$ & 42.04 & 53.45 & 66.34 \\
\hline $\mathbf{T}_{31}$ & D3+Control & 34.26 & 41.81 & 50.16 \\
\hline $\mathbf{T}_{32}$ & $\mathrm{D} 3+\mathrm{GA}_{3}(25 \mathrm{ppm})$ & 40.98 & 48.70 & 58.83 \\
\hline $\mathbf{T}_{33}$ & $\mathrm{D} 3+\mathrm{GA}_{3}(50 \mathrm{ppm})$ & 42.57 & 52.15 & 62.00 \\
\hline $\mathbf{T}_{34}$ & D3 +Brassinosteroids $(0.5 \mu \mathrm{M})$ & 40.73 & 48.81 & 58.56 \\
\hline $\mathbf{T}_{35}$ & D3 +Brassinosteroids $(1.0 \mu \mathrm{M})$ & 41.58 & 51.22 & 58.94 \\
\hline $\mathbf{T}_{36}$ & D3 + Boron (100ppm) & 41.72 & 50.54 & 58.88 \\
\hline $\mathbf{T}_{37}$ & D3 + Molybdenum $(2.0 \mu \mathrm{mol} / \mathrm{l})$ & 34.40 & 44.18 & 52.71 \\
\hline $\mathbf{T}_{38}$ & $\mathrm{D} 3+\mathrm{GA}_{3}(25 \mathrm{ppm})+$ Boron $(100 \mathrm{ppm})$ & 45.51 & 54.51 & 68.81 \\
\hline $\mathbf{T}_{39}$ & $\mathrm{D} 3+\mathrm{GA}_{3}(50 \mathrm{ppm})+$ Boron(100ppm) & 45.76 & 57.88 & 73.58 \\
\hline $\mathbf{T}_{40}$ & $\mathrm{D} 3+\mathrm{GA}_{3}(25 \mathrm{ppm})+$ Molybdenum $(2.0 \mu \mathrm{mol} / \mathrm{l})$ & 44.24 & 53.89 & 68.10 \\
\hline $\mathbf{T}_{41}$ & $\mathrm{D} 3+\mathrm{GA}_{3}(50 \mathrm{ppm})+$ Molybdenum $(2.0 \mu \mathrm{mol} / \mathrm{l})$ & 44.59 & 54.52 & 66.86 \\
\hline $\mathbf{T}_{42}$ & D3 +Brassinosteroids $(0.5 \mu \mathrm{M})+$ Boron $(100 \mathrm{ppm})$ & 43.58 & 53.30 & 66.08 \\
\hline $\mathbf{T}_{43}$ & $\mathrm{D} 3+$ Brassinosteroids $(1.0 \mu \mathrm{M})+$ Boron $(100 \mathrm{ppm})$ & 43.54 & 54.59 & 67.87 \\
\hline $\mathbf{T}_{44}$ & D3+Brassinosteroids $(0.5 \mu \mathrm{M})+$ Molybdenum $(2.0 \mu \mathrm{mol} / \mathrm{l})$ & 41.70 & 51.89 & 66.04 \\
\hline \multirow[t]{3}{*}{$\mathbf{T}_{45}$} & D3 +Brassinosteroids $(1.0 \mu \mathrm{M})+$ Molybdenum $(2.0 \mu \mathrm{mol} / \mathrm{l})$ & 42.22 & 52.85 & 65.92 \\
\hline & $\mathrm{SEm} \pm$ & 1.24 & 1.74 & 2.27 \\
\hline & C.D. at $5 \%$ level & 3.51 & 4.91 & 6.42 \\
\hline
\end{tabular}


Table.3 Effect of various plant growth regulators, micronutrients and photothermal regimes on no. of primary branches, No. of flowers per cluster and days to flower initiation

\begin{tabular}{|c|c|c|c|c|}
\hline $\begin{array}{l}\text { Treat. } \\
\text { Symb. }\end{array}$ & Treatments & $\begin{array}{l}\text { No. of primary } \\
\text { branches/ plant }\end{array}$ & $\begin{array}{l}\text { No. of flowers per } \\
\text { cluster }\end{array}$ & $\begin{array}{l}\text { Days to flower } \\
\text { initiation }\end{array}$ \\
\hline $\mathbf{T}_{1}$ & D1 +Control & 4.37 & 3.80 & 43.57 \\
\hline $\mathbf{T}_{2}$ & $\mathrm{D} 1+\mathrm{GA}_{3}(25 \mathrm{ppm})$ & 5.17 & 4.40 & 43.09 \\
\hline $\mathbf{T}_{3}$ & $\mathrm{D} 1+\mathrm{GA}_{3}(50 \mathrm{ppm})$ & 5.10 & 4.60 & 42.08 \\
\hline $\mathbf{T}_{4}$ & D1 +Brassinosteroids $(0.5 \mu \mathrm{M})$ & 5.03 & 4.33 & 43.28 \\
\hline $\mathbf{T}_{5}$ & D1 +Brassinosteroids $(1.0 \mu \mathrm{M})$ & 5.37 & 4.33 & 42.31 \\
\hline$T_{6}$ & D1 + Boron (100ppm) & 5.40 & 4.87 & 42.71 \\
\hline $\mathbf{T}_{7}$ & $\mathrm{D} 1+$ Molybdenum $(2.0 \mu \mathrm{mol} / \mathrm{l})$ & 5.50 & 4.13 & 43.38 \\
\hline $\mathbf{T}_{8}$ & $\mathrm{D} 1+\mathrm{GA}_{3}(25 \mathrm{ppm})+$ Boron $(100 \mathrm{ppm})$ & 5.60 & 4.80 & 38.32 \\
\hline $\mathbf{T}_{\mathbf{9}}$ & $\mathrm{D} 1+\mathrm{GA}_{3}(50 \mathrm{ppm})+$ Boron $(100 \mathrm{ppm})$ & 5.67 & 4.93 & 37.59 \\
\hline $\mathbf{T}_{10}$ & $\mathrm{D} 1+\mathrm{GA}_{3}(25 \mathrm{ppm})+$ Molybdenum $(2.0 \mu \mathrm{mol} / \mathrm{l})$ & 5.00 & 4.33 & 40.20 \\
\hline $\mathbf{T}_{11}$ & $\mathrm{D} 1+\mathrm{GA}_{3}(50 \mathrm{ppm})+$ Molybdenum $(2.0 \mu \mathrm{mol} / \mathrm{l})$ & 4.93 & 4.20 & 39.83 \\
\hline $\mathbf{T}_{12}$ & D1 +Brassinosteroids $(0.5 \mu \mathrm{M})+$ Boron(100ppm) & 5.20 & 4.73 & 39.51 \\
\hline $\mathbf{T}_{13}$ & $\mathrm{D} 1+$ Brassinosteroids $(1.0 \mu \mathrm{M})+$ Boron $(100 \mathrm{ppm})$ & 4.90 & 4.60 & 41.68 \\
\hline $\mathbf{T}_{14}$ & $\mathrm{D} 1+$ Brassinosteroids $(0.5 \mu \mathrm{M})+$ Molybdenum $(2.0 \mu \mathrm{mol} / \mathrm{l})$ & 4.77 & 4.20 & 41.29 \\
\hline $\mathbf{T}_{15}$ & $\mathrm{D} 1+$ Brassinosteroids $(1.0 \mu \mathrm{M})+$ Molybdenum $(2.0 \mu \mathrm{mol} / \mathrm{l})$ & 4.90 & 3.93 & 41.12 \\
\hline $\mathbf{T}_{16}$ & D2 +Control & 3.97 & 3.50 & 45.69 \\
\hline $\mathbf{T}_{17}$ & $\mathrm{D} 2+\mathrm{GA}_{3}(25 \mathrm{ppm})$ & 4.57 & 4.10 & 43.41 \\
\hline $\mathbf{T}_{18}$ & $\mathrm{D} 2+\mathrm{GA}_{3}(50 \mathrm{ppm})$ & 4.60 & 4.57 & 42.43 \\
\hline$T_{19}$ & $\mathrm{D} 2+$ Brassinosteroids $(0.5 \mu \mathrm{M})$ & 5.00 & 4.03 & 45.45 \\
\hline $\mathbf{T}_{20}$ & $\mathrm{D} 2+$ Brassinosteroids $(1.0 \mu \mathrm{M})$ & 4.57 & 4.07 & 42.51 \\
\hline $\mathbf{T}_{21}$ & $\mathrm{D} 2+$ Boron $(100 \mathrm{ppm})$ & 4.73 & 4.70 & 42.74 \\
\hline $\mathbf{T}_{22}$ & $\mathrm{D} 2+$ Molybdenum $(2.0 \mu \mathrm{mol} / \mathrm{l})$ & 5.03 & 3.90 & 44.24 \\
\hline $\mathbf{T}_{23}$ & $\mathrm{D} 2+\mathrm{GA}_{3}(25 \mathrm{ppm})+$ Boron $(100 \mathrm{ppm})$ & 5.00 & 4.57 & 38.46 \\
\hline $\mathbf{T}_{24}$ & $\mathrm{D} 2+\mathrm{GA}_{3}(50 \mathrm{ppm})+$ Boron $(100 \mathrm{ppm})$ & 5.57 & 4.77 & 37.76 \\
\hline $\mathbf{T}_{25}$ & $\mathrm{D} 2+\mathrm{GA}_{3}(25 \mathrm{ppm})+$ Molybdenum $(2.0 \mu \mathrm{mol} / \mathrm{l})$ & 4.63 & 3.73 & 40.33 \\
\hline $\mathbf{T}_{26}$ & $\mathrm{D} 2+\mathrm{GA}_{3}(50 \mathrm{ppm})+$ Molybdenum $(2.0 \mu \mathrm{mol} / \mathrm{l})$ & 4.53 & 4.27 & 40.09 \\
\hline $\mathbf{T}_{27}$ & D2 +Brassinosteroids $(0.5 \mu \mathrm{M})+$ Boron $(100 \mathrm{ppm})$ & 5.53 & 4.57 & 39.56 \\
\hline $\mathbf{T}_{28}$ & $\mathrm{D} 2+\operatorname{Brassinosteroids}(1.0 \mu \mathrm{M})+$ Boron $(100 \mathrm{ppm})$ & 4.57 & 4.57 & 41.72 \\
\hline $\mathbf{T}_{29}$ & $\mathrm{D} 2+$ Brassinosteroids $(0.5 \mu \mathrm{M})+$ Molybdenum $(2.0 \mu \mathrm{mol} / \mathrm{l})$ & 5.10 & 3.97 & 41.40 \\
\hline $\mathbf{T}_{30}$ & D2+Brassinosteroids $(1.0 \mu \mathrm{M})+$ Molybdenum $(2.0 \mu \mathrm{mol} / \mathrm{l})$ & 4.53 & 3.70 & 41.09 \\
\hline $\mathbf{T}_{31}$ & D3+Control & 3.63 & 3.23 & 45.89 \\
\hline $\mathbf{T}_{32}$ & $\mathrm{D} 3+\mathrm{GA}_{3}(25 \mathrm{ppm})$ & 4.50 & 3.67 & 43.65 \\
\hline $\mathbf{T}_{33}$ & $\mathrm{D} 3+\mathrm{GA}_{3}(50 \mathrm{ppm})$ & 4.30 & 4.23 & 42.50 \\
\hline $\mathbf{T}_{34}$ & D3 +Brassinosteroids $(0.5 \mu \mathrm{M})$ & 4.90 & 3.67 & 45.59 \\
\hline $\mathbf{T}_{35}$ & D3 +Brassinosteroids $(1.0 \mu \mathrm{M})$ & 4.63 & 3.80 & 42.68 \\
\hline $\mathbf{T}_{36}$ & D3 + Boron (100ppm) & 4.40 & 4.37 & 42.89 \\
\hline $\mathbf{T}_{37}$ & D3 + Molybdenum $(2.0 \mu \mathrm{mol} / \mathrm{l})$ & 4.63 & 3.53 & 45.71 \\
\hline $\mathbf{T}_{38}$ & D3+ $\mathrm{GA}_{3}(25 \mathrm{ppm})+$ Boron (100ppm) & 4.57 & 4.30 & 38.57 \\
\hline $\mathbf{T}_{39}$ & $\mathrm{D} 3+\mathrm{GA}_{3}(50 \mathrm{ppm})+$ Boron(100ppm) & 4.13 & 4.43 & 37.88 \\
\hline $\mathbf{T}_{40}$ & $\mathrm{D} 3+\mathrm{GA}_{3}(25 \mathrm{ppm})+$ Molybdenum(2.0 $\left.\mu \mathrm{mol} / \mathrm{l}\right)$ & 4.30 & 3.63 & 40.57 \\
\hline $\mathbf{T}_{41}$ & $\mathrm{D} 3+\mathrm{GA}_{3}(50 \mathrm{ppm})+$ Molybdenum(2.0 $\left.\mu \mathrm{mol} / \mathrm{l}\right)$ & 4.20 & 3.70 & 40.40 \\
\hline $\mathbf{T}_{42}$ & D3 +Brassinosteroids $(0.5 \mu \mathrm{M})+$ Boron(100ppm) & 4.60 & 4.23 & 39.79 \\
\hline $\mathbf{T}_{43}$ & 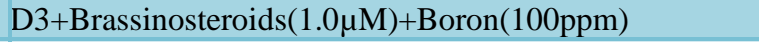 & 4.03 & 4.23 & 41.87 \\
\hline $\mathbf{T}_{44}$ & D3+Brassinosteroids $(0.5 \mu \mathrm{M})+$ Molybdenum $(2.0 \mu \mathrm{mol} / \mathrm{l})$ & 4.50 & 3.63 & 41.72 \\
\hline \multirow[t]{3}{*}{$\mathbf{T}_{45}$} & D3+Brassinosteroids $(1.0 \mu \mathrm{M})+$ Molybdenum $(2.0 \mu \mathrm{mol} / \mathrm{l})$ & 4.20 & 3.37 & 41.04 \\
\hline & $\mathrm{SEm} \pm$ & 0.26 & 0.20 & 1.23 \\
\hline & C.D. at $5 \%$ level & 0.72 & 0.55 & 3.47 \\
\hline
\end{tabular}


Table.4 Effect of various plant growth regulators, micronutrients and photothermal regimes on days to $50 \%$ flowering, days to first picking and fruit yield (Kg/Plot)

\begin{tabular}{|c|c|c|c|c|}
\hline $\begin{array}{l}\text { Treat. } \\
\text { Symb. }\end{array}$ & Treatments & $\begin{array}{l}\text { Days to } 50 \% \\
\text { flowering }\end{array}$ & $\begin{array}{c}\text { Days to first } \\
\text { picking }\end{array}$ & $\begin{array}{c}\text { Fruit yield } \\
\text { (Kg/plot) }\end{array}$ \\
\hline $\mathrm{T}_{1}$ & D1 +Control & 48.39 & 62.38 & 23.40 \\
\hline $\mathbf{T}_{2}$ & $\mathrm{D} 1+\mathrm{GA}_{3}(25 \mathrm{ppm})$ & 48.36 & 58.30 & 30.37 \\
\hline $\mathbf{T}_{3}$ & $\mathrm{D} 1+\mathrm{GA}_{3}(50 \mathrm{ppm})$ & 47.29 & 56.09 & 31.26 \\
\hline $\mathbf{T}_{4}$ & D1 +Brassinosteroids $(0.5 \mu \mathrm{M})$ & 48.08 & 61.21 & 27.11 \\
\hline $\mathbf{T}_{5}$ & D1 +Brassinosteroids $(1.0 \mu \mathrm{M})$ & 47.48 & 58.26 & 33.29 \\
\hline $\mathbf{T}_{6}$ & D1 + Boron (100ppm) & 47.70 & 57.81 & 30.59 \\
\hline $\mathbf{T}_{7}$ & $\mathrm{D} 1+$ Molybdenum $(2.0 \mu \mathrm{mol} / \mathrm{l})$ & 48.19 & 62.21 & 23.82 \\
\hline $\mathbf{T}_{8}$ & $\mathrm{D} 1+\mathrm{GA}_{3}(25 \mathrm{ppm})+$ Boron (100ppm) & 42.20 & 55.34 & 43.25 \\
\hline $\mathbf{T}_{9}$ & $\mathrm{D} 1+\mathrm{GA}_{3}(50 \mathrm{ppm})+\operatorname{Boron}(100 \mathrm{ppm})$ & 40.26 & 55.19 & 44.02 \\
\hline $\mathbf{T}_{10}$ & $\mathrm{D} 1+\mathrm{GA}_{3}(25 \mathrm{ppm})+$ Molybdenum $(2.0 \mu \mathrm{mol} / \mathrm{l})$ & 47.05 & 57.32 & 41.04 \\
\hline $\mathbf{T}_{11}$ & $\mathrm{D} 1+\mathrm{GA}_{3}(50 \mathrm{ppm})+$ Molybdenum $(2.0 \mu \mathrm{mol} / \mathrm{l})$ & 45.43 & 56.29 & 41.21 \\
\hline $\mathbf{T}_{12}$ & D1 +Brassinosteroids $(0.5 \mu \mathrm{M})+$ Boron(100ppm) & 45.26 & 56.17 & 41.18 \\
\hline $\mathbf{T}_{13}$ & D1 +Brassinosteroids $(1.0 \mu \mathrm{M})+$ Boron(100ppm) & 47.41 & 58.03 & 43.55 \\
\hline $\mathbf{T}_{14}$ & D1+Brassinosteroids $(0.5 \mu \mathrm{M})+$ Molybdenum $(2.0 \mu \mathrm{mol} / \mathrm{l})$ & 47.30 & 57.34 & 36.32 \\
\hline $\mathbf{T}_{15}$ & D1+Brassinosteroids $(1.0 \mu \mathrm{M})+$ Molybdenum $(2.0 \mu \mathrm{mol} / \mathrm{l})$ & 47.19 & 57.19 & 36.61 \\
\hline $\mathbf{T}_{16}$ & D2 +Control & 52.14 & 66.23 & 21.33 \\
\hline $\mathbf{T}_{17}$ & $\mathrm{D} 2+\mathrm{GA}_{3}(25 \mathrm{ppm})$ & 50.46 & 63.41 & 31.15 \\
\hline $\mathbf{T}_{18}$ & $\mathrm{D} 2+\mathrm{GA}_{3}(50 \mathrm{ppm})$ & 48.23 & 63.36 & 32.59 \\
\hline$T_{19}$ & $\mathrm{D} 2+$ Brassinosteroids $(0.5 \mu \mathrm{M})$ & 50.61 & 64.67 & 26.10 \\
\hline $\mathbf{T}_{20}$ & $\mathrm{D} 2+$ Brassinosteroids $(1.0 \mu \mathrm{M})$ & 48.43 & 63.30 & 31.69 \\
\hline $\mathbf{T}_{21}$ & D2 + Boron (100ppm) & 48.47 & 61.37 & 25.98 \\
\hline $\mathbf{T}_{22}$ & $\mathrm{D} 2+$ Molybdenum $(2.0 \mu \mathrm{mol} / \mathrm{l})$ & 51.27 & 65.08 & 22.74 \\
\hline $\mathbf{T}_{23}$ & $\mathrm{D} 2+\mathrm{GA}_{3}(25 \mathrm{ppm})+$ Boron (100ppm) & 44.23 & 57.33 & 41.14 \\
\hline $\mathbf{T}_{24}$ & $\mathrm{D} 2+\mathrm{GA}_{3}(50 \mathrm{ppm})+$ Boron(100ppm) & 44.15 & 55.83 & 43.00 \\
\hline $\mathbf{T}_{25}$ & $\mathrm{D} 2+\mathrm{GA}_{3}(25 \mathrm{ppm})+$ Molybdenum(2.0 $\left.\mu \mathrm{mol} / \mathrm{l}\right)$ & 45.38 & 59.30 & 39.64 \\
\hline $\mathbf{T}_{26}$ & $\mathrm{D} 2+\mathrm{GA}_{3}(50 \mathrm{ppm})+$ Molybdenum $(2.0 \mu \mathrm{mol} / \mathrm{l})$ & 45.18 & 58.33 & 40.27 \\
\hline $\mathbf{T}_{27}$ & $\mathrm{D} 2+$ Brassinosteroids $(0.5 \mu \mathrm{M})+$ Boron $(100 \mathrm{ppm})$ & 45.10 & 58.19 & 39.01 \\
\hline $\mathbf{T}_{28}$ & $\mathrm{D} 2+$ Brassinosteroids $(1.0 \mu \mathrm{M})+$ Boron $(100 \mathrm{ppm})$ & 47.28 & 61.02 & 39.81 \\
\hline$T_{29}$ & D2+Brassinosteroids $(0.5 \mu \mathrm{M})+$ Molybdenum $(2.0 \mu \mathrm{mol} / \mathrm{l})$ & 47.32 & 60.40 & 34.47 \\
\hline $\mathbf{T}_{30}$ & D2+Brassinosteroids $(1.0 \mu \mathrm{M})+$ Molybdenum $(2.0 \mu \mathrm{mol} / \mathrm{l})$ & 47.23 & 60.24 & 35.46 \\
\hline $\mathbf{T}_{31}$ & D3+Control & 52.53 & 65.32 & 19.18 \\
\hline $\mathbf{T}_{32}$ & $\mathrm{D} 3+\mathrm{GA}_{3}(25 \mathrm{ppm})$ & 50.62 & 61.32 & 29.04 \\
\hline $\mathbf{T}_{33}$ & $\mathrm{D} 3+\mathrm{GA}_{3}(50 \mathrm{ppm})$ & 48.40 & 60.33 & 33.69 \\
\hline $\mathbf{T}_{34}$ & D3 + Brassinosteroids $(0.5 \mu \mathrm{M})$ & 50.68 & 61.60 & 24.70 \\
\hline $\mathbf{T}_{35}$ & D3 + Brassinosteroids $(1.0 \mu \mathrm{M})$ & 48.54 & 59.21 & 29.04 \\
\hline $\mathbf{T}_{36}$ & D3 + Boron (100ppm) & 48.58 & 59.37 & 24.91 \\
\hline $\mathbf{T}_{37}$ & D3 + Molybdenum $(2.0 \mu \mathrm{mol} / \mathrm{l})$ & 55.41 & 64.38 & 20.31 \\
\hline $\mathbf{T}_{38}$ & $\mathrm{D} 3+\mathrm{GA}_{3}(25 \mathrm{ppm})+$ Boron (100ppm) & 50.45 & 60.15 & 39.28 \\
\hline $\mathbf{T}_{39}$ & $\mathrm{D} 3+\mathrm{GA}_{3}(50 \mathrm{ppm})+$ Boron(100ppm) & 50.31 & 60.09 & 41.65 \\
\hline $\mathbf{T}_{40}$ & $\mathrm{D} 3+\mathrm{GA}_{3}(25 \mathrm{ppm})+$ Molybdenum(2.0 $\left.\mu \mathrm{mol} / \mathrm{l}\right)$ & 51.46 & 61.24 & 37.77 \\
\hline $\mathbf{T}_{41}$ & $\mathrm{D} 3+\mathrm{GA}_{3}(50 \mathrm{ppm})+$ Molybdenum $(2.0 \mu \mathrm{mol} / \mathrm{l})$ & 51.21 & 61.10 & 40.83 \\
\hline $\mathbf{T}_{42}$ & D3 +Brassinosteroids $(0.5 \mu \mathrm{M})+$ Boron $(100 \mathrm{ppm})$ & 45.42 & 55.33 & 38.93 \\
\hline $\mathbf{T}_{43}$ & D3+Brassinosteroids $(1.0 \mu \mathrm{M})+$ Boron(100ppm) & 47.43 & 52.22 & 37.44 \\
\hline $\mathbf{T}_{44}$ & D3+Brassinosteroids $(0.5 \mu \mathrm{M})+$ Molybdenum $(2.0 \mu \mathrm{mol} / \mathrm{l})$ & 47.56 & 52.41 & 30.65 \\
\hline \multirow[t]{3}{*}{$\mathbf{T}_{45}$} & D3+Brassinosteroids $(1.0 \mu \mathrm{M})+$ Molybdenum $(2.0 \mu \mathrm{mol} / \mathrm{l})$ & 47.47 & 52.40 & 32.00 \\
\hline & $\mathrm{SEm} \pm$ & 1.43 & 1.57 & 2.02 \\
\hline & C.D. at $5 \%$ level & 4.04 & 4.43 & 5.71 \\
\hline
\end{tabular}




\section{Number of primary branches per plant}

The maximum number of primary branches per plant was noted under $D_{1} T_{9}$ (5.67) which were transplanted on $15^{\text {th }}$ November with the foliar application of $\mathrm{GA}_{3}(50 \mathrm{ppm})+$ Boron (100ppm) followed by $\mathrm{D}_{1} \mathrm{~T}_{8}(5.60)$ while the minimum number of primary branches was noted under control- $\mathrm{D}_{3} \mathrm{~T}_{31}$ (3.63). The increase in number of primary branches may be due to promoting effect of GA3 in protein synthesis which consequently enhances biomass production of vegetative parts and their content as reported by Khan et al., (2006). These results are similar to findings of Dhakar and Singh (2015), Meena et al., (2015), Akand et al., (2016) and Jakhar et al., (2018) (Table 2).

\section{Number of flower per cluster}

The highest number of flowers per cluster was observed in $\mathrm{D}_{1} \mathrm{~T}_{9}$ (4.93) followed by $\mathrm{D}_{1} \mathrm{~T}_{6}$ (4.87) while the minimum number of flowers per cluster was observed in $\mathrm{D}_{3} \mathrm{~T}_{31}$ (3.23). Number of flowers per cluster mainly depends on number of branches plant ${ }^{-1}$. As the GA3 resulted in maximum number of branches per plant therefore number of flower cluster $^{-1}$ was also increased. This result is in accordance with the findings of Islam (2015), Meena et al., (2015)

\section{Days to flower initiation}

The maximum days to flower initiation was noted under in $\mathrm{D}_{3} \mathrm{~T}_{31}$ (45.89) which was control and transplanted on $15^{\text {th }}$ December followed by $\mathrm{D}_{3} \mathrm{~T}_{37}$ (45.71) while the minimum days to flower initiation was noted under in $\mathrm{D}_{1} \mathrm{~T}_{9}$ (37.59) which were transplanted on $15^{\text {th }}$ November with the foliar application of $\mathrm{GA}_{3}$ (50ppm) + Boron $(100 \mathrm{ppm})$. The probable reason for increase in days to flower initiation is well known to increase hydrolysis of starch and sucrose into glucose and fructose, which were utilized by the flowers for floret opening and boron showed the highest $\mathrm{P}$ and $\mathrm{K}$ uptake at the pre flowering $(\mathrm{P} \& \mathrm{~K})$ and flowering $(\mathrm{P}$ only) stages. This was attributed to the increased photosynthesis and effective translocation of photosynthates. These results are similar to the findings of Gupta and Chakrabarty (2013).

\section{Days to 50\% flowering}

The maximum days to 50 percent flowering was recorded in $\mathrm{D}_{3} \mathrm{~T}_{37}$ (55.41) followed by $\mathrm{D}_{3} \mathrm{~T}_{31}$ (52.53) while the minimum days to 50 percent flowering was recorded in $\mathrm{D}_{1} \mathrm{~T}_{9}$ (40.26). The findings are in close harmony with the result of Jakhar et al., (2018) and Kumar et al., (2018) (Table 3).

\section{Days to first picking}

The maximum days to first picking was noted under $\mathrm{D}_{2} \mathrm{~T}_{16}$ (66.23) followed by $\mathrm{D}_{3} \mathrm{~T}_{31}$ (65.32) while the minimum days to first picking was noted under $\mathrm{D}_{3} \mathrm{~T}_{43}(52.22)$ in first year. This might be due to that $\mathrm{GA}_{3}$ may enhance source to sink relationship, accumulation of photosynthates and efficient utilization of food reserves for the development of fruit and also due to role of boron in flower development, pollen germination, fertilization and fruit abscission. Foliar spray of borax at 60 and 90 DAT closely synchronized with fruit development as it plays role in translocation of carbohydrates to developing fruits.

\section{Fruit Yield (Kg/Plot)}

The highest fruit yield per plot was recorded in $\mathrm{D}_{1} \mathrm{~T}_{9}$ (44.02) followed by $\mathrm{D}_{1} \mathrm{~T}_{13}$ (43.55) while the lowest fruit yield per plot was observed in $\mathrm{D}_{3} \mathrm{~T}_{31}$ (19.18). this may be due to $\mathrm{GA}_{3}$ are growth motivating substance which improve the plant growth and development and also increase the fruit set as well as 
improve yield per plant which ultimately increases yield per plot and increment of paranchymatous cells in pericarp and thickness of vascular tissue as a result of boron treatment led to increase the thickness of pericarp or fruit wall which finally increased the yield. The result is proximate to that of the Khan et al., (2006), Meena et al., (2015) and Akand et al., (2016).

On the basis of this investigation, it is concluded that the treatment $\mathrm{GA}_{3}(50 \mathrm{ppm})+$ Boron (100ppm) $\left(\mathrm{D}_{1} \mathrm{~T}_{9}\right)$ responded well in terms of growth and yield parameters. Application of foliar spray of the treatment $\mathrm{GA}_{3}(50 \mathrm{ppm})+$ Boron $(100 \mathrm{ppm})\left(\mathrm{D}_{1} \mathrm{~T}_{9}\right)$ were recorded significantly higher morphological growth (viz., plant height, number of primary branches per plant, number of flower per cluster), phenological parameters (viz., days to flower initiation, days to $50 \%$ flowering, days to first picking) and fruit yield (Kg/plot).

\section{References}

Abd El-Gawad HG and Osman HS. 2014. Effect of Exogenous Application of Boric Acid and Seaweed Extract on Growth, Biochemical Content and Yield of Eggplant. Journal of Horticultural Science \& Ornamental Plants 6 (3): 133-143.

Akand MH, Mazed HEMK, BhagatSK ,Moonmoon JF and Moniruzzaman $\mathrm{M}$. 2016. Growth and yield of tomato as influenced by potassium and gibberellic acid. Bulletin of the institute of tropical agriculture 39: 83-94.

Bajguz A and Hayat S. 2009.Effects of brassinosteroids on the plant responses to environmental stresses. Plant Physiology and Biochemistry 47(1):1-8.

Cao S, Xu Q, Cao Y, Qian K, An K, Zhu Y, BinzengH, Zhao H, Kuai B. 2005. Loss-offunction mutations in DET2gene lead to an enhanced resistance to oxidative stress in Arabidopsis. - Plant Physiology 123: 57-66.

Chauhan DVS. 1981. Vegetable Production in India, Ram Prasad and Sons, Agriculture Publishing House Agra.
Dhakar S, Singh Y. 2015. Studies on the effect of inorganic fertilizers and plant growth regulator on growth and yield of brinjal (Solanum melongena L.). Indian Journal of Basic and. Applied Medical Research 1(2):27-39.

Gupta R and Chakrabarty SK. 2013. Gibberellic acid in plant still a mystery unresolved. Plant signaling and behavior 8(9): 1-6.

Hayat S, Alyemeni MN, Hasan SA. 2012. Foliar spray of brassinosteroid enhances yield and quality of Solanum lycopersicum under cadmium stress. Saudi Journal of Biological Sciences 19: 325-335.

Islam S, Islam MM, Siddik MA, Afsana N, Rabin MH, Hossain MD, Parvin S. 2017. Variation in Growth and Yield of Tomato at Different Transplanting Time. International Journal of Scientific and Research Publications 7(2): 142-145.

Jakhar D, Thaneshwari , Nain S and Jakhar. 2018. Effect of Plant Growth Regulator on Growth, Yield \& Quality of Tomato (Solanum lycopericum) Cultivar 'Shivaji' under Punjab Condition. International Journal of Current Microbiology and Applied Science 7(6): 2630-2636.

Khan MMA, Gautam FM, Siddiqui MH, Naeem $M$ and Khan MN. 2006. Effect of gibberellic acid spray on performance of tomato. Turkish Journal of Biology 30: 1116.

KrishnaP. 2003. Brassinosteroid-Mediated Stress Responses. Journal of Plant Growth Regulation 22(4):289-297.

Kumar A, Biswas TK, Singh N and Dr. Lal EP. 2014. Effect of Gibberellic Acid on Growth, Quality and Yield of Tomato (Lycopersiconesculentum Mill.). Journal of Agriculture and Veterinary Science 7(7): 28-30.

Meena SS, Dhaka RS and Jalwania R. 2005. Economics of plant growth regulators in brinjal (Solanum melongena L.) under semi-arid condition of Rajasthan. Agricultural Science Digest 25(4): 248 250.

Mohamed AE, Rashed MN, Mofty A. 2003. Assessment of essential and toxic elements in some kinds of vegetables. Ecotoxicology and Environmental Safety 55(3): 251-60. 
Montoya T, Nomura T, Farrar K, Kaneta T, Yokota T and Bishop GJ. 2002. Cloning the tomato Curl3 gene highlights the putative dual role of the leucine-rich repeat receptor kinase tBRI1/SR160 in plant steroid hormone and peptide hormone signaling. Plant Cell 14: 3163- 3176.

Nakashita H, Yasuda M, Nitta T , Asami T, Fujioka S, Arai Y, Sekimata K, Takatsuto S, Yamaguchi I and Yoshida S. 2003. Brassinosteroid functions in a broad range of disease resistance in tobacco and rice. The Plant Journal 33: 887-898.

Nath P, Velayudhan S and Singh DP. 1987. Vegetable for the Tropical Region. Indian Council of Agricultural Research, New Delhi 23-24.

NHB. 2018. National Horticulture Board, nhb.gov.in.

Nothmann J and Koller D.1973. Morphologenetic effects of low temperature stress on flowering of eggplant (Solanum melongena L.). Israel Journal of Botany22: 231-235.

Ogweno JO, Song XS, Shi K, Wen, Hu H, Mao WH, Zhou YH, Yu JQ and Nogue's S. 2008. Brassinosteroids Alleviate HeatInduced Inhibition of Photosynthesis by Increasing Carboxylation Efficiency and Enhancing Antioxidant Systems in Lycopersicon esculentum. Journal of Plant Growth Regulation 27:49-57.

Raigón MD, Prohens J, Muñoz-Falcón JE, Nuez F. 2008. Comparison of eggplant landraces and commercial varieties for fruit content of phenolics, minerals, dry matter and protein. Journal of Food Composition and Analysis 21(5):370-376.

Roychowdhury Rand Tah J. 2011. Differential response by different parts of Solanummelongena 1 . for heavy metal accumulation. Plant Sciences Feed 1(6): 8083.

Sabatino L, D’Anna F, Lapichino G, Moncada A, D'Anna E and Pasquale CD. 2019. Interactive effects of genotype and molybdenum supply on yield and overall fruit quality of tomato. Frontiers in Plant Science 9:1922 p 1-10.

Sanchez-Castillo CP, EnglystHN, Hudson GJ, Lara JJ, Solano ML, Munguia JL and James WP. 1999. The non-starch polysaccharide content of Mexican foods. Journal of Food Composition and Analysis 12(4): 293-314.

Sasse JM. 2003. Physilogical actions of brassinosteroids: an update. Journal of Plant Growth Regulation 22: 276-288.

Siddiky MA, Halder NK, Islam Z, Begam RA and Masud MM. 2007. Performance of Brinjal as influenced by boron and molybdenum. Asian Journal of Plant Sciences 6(2): 389393.

Tindall HD. 1978. Commercial vegetable growing. Oxford University press, London p. 129.

USDA (United States Department of Agriculture). 2014. USDA National Nutrient Database for Standard Reference. http://www.nal.usda.gov/fnic/foodcomp/se arch.

Vavilov NI 1928. Proceedings $5^{\text {th }}$ International Congress of Genetics, New York 42-369.

Zeaven AC and Zhukovsky PM. 1975. Dictionary of cultivated plants and their centre of diversity. Wageningen, Netherlands p 219.

\section{How to cite this article:}

Diksha Manaware and Sharma, D.P. 2020. Effect of Different Plant Growth Regulators, Micronutrients and Photothermal Regimes on Growth, Phenological and Yield Characters of Brinjal (Solanum melongena L.). Int.J.Curr.Microbiol.App.Sci. 9(10): 3241-3249. doi: https://doi.org/10.20546/ijcmas.2020.910.387 